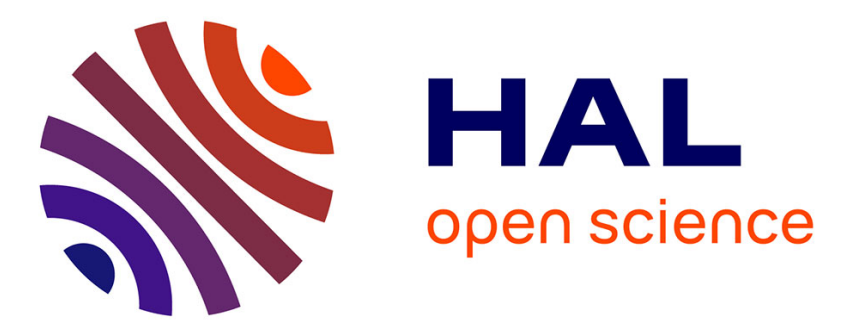

\title{
Développement de la référence à soi chez une enfant de $1 ; 05$ à 3;00: de l'influence de l'input à la reconstruction du système
}

\author{
Stéphanie Caët
}

\section{- To cite this version:}

Stéphanie Caët. Développement de la référence à soi chez une enfant de 1;05 à 3;00: de l'influence de l'input à la reconstruction du système. Journal of French Language Studies, 2012, pp.77-93. 10.1017/S0959269511000597 . hal-00657834

\section{HAL Id: hal-00657834 https://hal.science/hal-00657834}

Submitted on 25 Jan 2012

HAL is a multi-disciplinary open access archive for the deposit and dissemination of scientific research documents, whether they are published or not. The documents may come from teaching and research institutions in France or abroad, or from public or private research centers.
L'archive ouverte pluridisciplinaire HAL, est destinée au dépôt et à la diffusion de documents scientifiques de niveau recherche, publiés ou non, émanant des établissements d'enseignement et de recherche français ou étrangers, des laboratoires publics ou privés. 


\title{
Développement de la référence à soi
}

\author{
chez une enfant de 1;05 à 3;00 : \\ de l'influence de l'input à la reconstruction du système \\ Stéphanie CAET \\ Université Sorbonne Nouvelle - Paris 3
}

\begin{abstract}
Cette étude adopte une approche à la fois fonctionnaliste et usage-based de l'acquisition du langage pour rendre compte des formes non conventionnelles de référence à soi en position sujet chez une enfant francophone, Anaé, entre 1;05 et 3;00. D'une part, nos analyses quantitatives et qualitatives révèlent la présence, après 2 ans, d'un paradigme de verbes à valeur modale pour lesquels la référence à soi en position sujet peut être implicite. Pour ces verbes, comme pour tous les verbes avant 2 ans, un sujet grammatical est essentiellement produit dans des situations où l'enfant exprime un positionnement intersubjectif. D'autre part, la production de formes non conventionnelles met en évidence la création d'associations entre des formes et des contextes interactionnels spécifiques. L'analyse du langage adressé à l'enfant suggère que ces associations trouvent notamment leur origine dans le langage de la mère.
\end{abstract}

\section{Mots CLES}

Acquisition du langage, référence à soi, formes non conventionnelles, constructions, input, grammaticalisation 


\section{INTRODUCTION}

Lorsque l'enfant entre dans le langage, ses premières productions reflètent à la fois les stratégies qu'il emploie pour exprimer des besoins communicationnels spécifiques (Ninio et Snow, 1996) et l'influence de la langue qui l'entoure, qu'il reconstruit progressivement (Tomasello, 2003). L'analyse détaillée de ses productions et en particulier de ses productions non conventionnelles (cf. Chiat, 1986) offre alors un accès privilégié au rôle que jouent ces deux facteurs dans la construction de son système linguistique.

Le développement de la référence à soi est un lieu propice à l'étude du rôle de l'input dans la reconstruction active du système par l'enfant. Les analyses de Budwig (1995, 1996) sur l'anglais montre en effet que les enfants produisent des structures $[m e+$ verbe] pour exprimer une agentivité contrastée, et qu'ils emploient $[I+$ verbe] dans des contextes où le référent sujet est faiblement agentif, comme c'est également le cas dans le langage des parents. Dans la continuité de ces travaux sur l'acquisition de l'anglais, la présente étude questionne le rôle de l'input sur le développement de la référence à soi en position sujet chez une enfant en cours d'acquisition d'un système linguistique différent : le français.

\subsection{La référence à soi en position sujet chez l'enfant francophone}

Lorsque l'enfant entre dans la mise en mots du soi en tant qu'agent ou expérient d'un procès, une grande variété de formes peut être produite en position sujet. Les analyses quantitatives et qualitatives conduites sur les données longitudinales de trois enfants francophones âgés de 1;08 à 3;00 par Brigaudiot, Morgenstern et Nicolas (1994) et Morgenstern $(1995,2006)$ ont 
montré qu'un enfant en acquisition du français peut, à une même période, produire des prédicats référant à lui-même avec des sujets non conventionnels (« [ə] veux », « moi veux », « tu veux », « il/i veut » ou « Léonard (i) veut ») et des sujets conventionnels (« je veux», « moi je veux»). Les prédicats peuvent également être produits sans sujet grammatical («veux»). Selon ces auteurs, cette diversité reflète la mise en place par l'enfant d'un système dans lequel a) un prédicat sans sujet ou précédé d'une voyelle préverbale (aussi appelée «filler»- Peters et Menn, 1993) ou d'un je, est employé dans l'expression du désir, de la volonté ou du projet, et b) $[m o i+(j e)+$ verbe $]$ ou le [prénom+verbe] sont produits lorsque l'enfant marque une distanciation, visà-vis de son interlocuteur avec moi, et vis-à-vis d'un soi passé ou représenté sur une photographie avec le prénom (cf. travaux sur l'image spéculaire de Zazzo, 1948). Quant à l'emploi de la deuxième ou troisième personne, phénomène rare (Chiat, 1986) mais parfois perçu comme une marque de confusion dans l'acquisition des pronoms personnels (Charney, 1980 ; Petitto, 1987 pour la LSF), il est analysé comme un emprunt du langage adulte pour mettre en mot l'exploit ou la bêtise (Morgenstern et Brigaudiot, 2004).

Ces études sur le français font écho aux analyses de cas longitudinales menées à la même période par Budwig (1995) en anglais, dont l'approche fonctionnaliste sémantique basée sur l'expression de l'agentivité rejoint l'approche énonciativiste adoptée dans la description des données francophones. Contrairement aux hypothèses générativistes qui analysent les formes non adultes produites par l'enfant comme le signe de l'immaturité de sa grammaire ou de ses limitations en production (cf. parmi de nombreuses études : Gerken, 1994 ; Rispoli, 2005), ces approches mettent donc l'accent 
sur la reconstruction active du système par l'enfant selon ses besoins communicationnels. Progressivement, ce système se grammaticalise. L'enfant ne compte plus uniquement sur l'adulte pour interpréter ses énoncés (Morgenstern, 1996) et produit donc de moins en moins de prédicats sans sujet grammatical ; les formes non conventionnelles disparaissent et d'autres marqueurs remplissent les fonctions pragmatiques que ces formes exprimaient.

\subsection{Le rôle de l'input dans le développement de l'auto-désignation}

Soulevant la question de l'influence du langage des parents sur le développement de la référence à soi chez l'enfant, Budwig (1996) a montré par la suite que l'association entre la structure $[I+$ verbe $]$ et un degré d'agentivité faible du référent sujet pouvait être recréée par les enfants à partir du langage des parents, chez qui le pronom personnel sujet $I$ est souvent associé à un référent non agentif.

L'hypothèse d'une influence de l'input sur les formes de référence à soi chez l'enfant, souvent proposée pour expliquer l'emploi du prénom (Wills, 1977 ; Chiat, 1981 ; Rabain-Jamin et Sabeau-Jouannet, 1989 ; Morgenstern, 2011), a récemment été testée par Kirjavainen, Theakston et Lieven (2009) dans le cadre des théories constructivistes (Tomasello, 2003 ; voir aussi l'introduction de ce numéro) pour rendre compte de la production des structures [me+verbe] comme «me do it» par les enfants anglophones. Cette étude transversale montre que de telles structures sont plus nombreuses chez des enfants dont les parents produisent eux-mêmes un grand nombre de séquences où le pronom me est suivi d'un prédicat, comme dans « let me do it ». La présence simultanée des formes conventionnelles et non 
conventionnelles dans le langage des enfants s'explique alors par l'identification parallèle des structures [I+verbe] et [me+verbe], qui entrent en compétition dans le lexique de l'enfant. Celui-ci choisira l'une ou l'autre selon la fréquence avec laquelle il les entend dans le langage des adultes, et selon le prédicat produit.

Cette hypothèse, cependant, ne semble rendre compte que partiellement du développement de la référence à soi en position sujet chez l'enfant francophone. En effet, si des constructions comme « laisse-moi faire » existent en français, rares sont les cas où le verbe est à l'infinitif dans les structures [moi+verbe] produites par les enfants francophones (ils sont absents des données analysées ici). Rares sont également les cas où moi en fin d'énoncé précède immédiatement un prédicat en début d'énoncé suivant, car les inversions sujet-verbe sont peu fréquentes en français oral (Gadet, 1989).

Aussi, l'approche sémantique de l'input adoptée par Budwig (1996) peut davantage nous intéresser pour le français: elle ne repose pas uniquement sur les caractéristiques formelles du langage adressé à l'enfant et de la langue en acquisition, et offre ainsi la possibilité de rendre compte du phénomène chez des enfants apprenant des langues différentes. Appliquée aux données du français, cette hypothèse permettrait d'expliquer pourquoi l'enfant francophone utilise la forme moi pour marquer son positionnement dans l'interaction (Morgenstern, 1995, 2006) : en français oral, ce marqueur permet en effet de mettre l'emphase sur le sujet lorsqu'il est employé dans des structures [moi $+j e+$ verbe], et de marquer l'opposition ou la comparaison avec d'autres référents. Ainsi, lorsque ces structures emphatiques sont employées par les parents dans les interactions adulte-enfant, leur forme et les 
contextes d'utilisation sont probablement suffisamment saillants pour que les enfants identifient rapidement leur fonction contrastive (cf. Clark et Clark, 1979) et les intègrent comme des marqueurs de positionnement intersubjectif, à une période où ils chercheraient dans la langue les formes leur permettant de marquer leur besoin croissant d'autonomie (Mahler, Pine et Bergman, 1975).

Nous suggérons ici que le croisement des approches fonctionnalistes et d'une approche basée sur l'usage du langage par les parents permet à la fois de mettre en évidence la systématicité des contextes discursifs et interactionnels dans lesquels certaines formes apparaissent en position sujet dans les énoncés de l'enfant, et de retracer l'origine dans l'input de ces paires forme-fonction. La coarticulation de ces approches offre ainsi la possibilité d'étudier le développement de la référence à soi en prenant en compte les connaissances linguistiques de l'enfant, ses intentions communicatives, mais aussi l'influence des propriétés formelles et fonctionnelles du langage qui lui est adressé.

\subsection{Objectifs de cette étude}

Dans cet article, nous analysons l'émergence et le développement de la référence à soi en position sujet dans le corpus d'Anaé (Morgenstern, 2009) entre $1 ; 05$ et 3;00 (voir le chapitre de présentation des corpus de ce numéro pour plus de détails sur Anaé et son développement linguistique).

A la suite des travaux de Budwig (1995) en anglais, et de Brigaudiot et al. (1994) et Morgenstern (1995; 2006) en français, nous montrons dans un premier temps que des formes spécifiques sont produites dans les énoncés où l'enfant réfère à elle-même selon ses intentions communicatives. Dans un 
deuxième temps, à la suite de Budwig (1996), nous analysons les productions non conventionnelles de référence à soi en position sujet au regard de formes comparables de référence à soi et à l'enfant utilisées par la mère, et des contextes dans lesquels ces formes sont employées.

\section{METHODOLOGIE ET ANALYSES PRELIMINAIRES}

\subsection{Méthodologie d'analyse}

Tous les énoncés contenant un prédicat verbal dans lesquels la référence à soi est ou pourrait être encodée par un sujet grammatical ont été relevés dans les énoncés d'Anaé lorsque celle-ci s'adresse à sa mère. Des éléments contextuels, mimo-gestuels et prosodiques ont permis d'identifier les énoncés dans lesquels la référence à soi n'est pas exprimée par un sujet grammatical ou dans lesquels elle est marquée par une forme non conventionnelle.

Sur l'ensemble des données (soit 16 heures d'enregistrement), Anaé produit 385 prédicats référant à elle (avec ou sans sujet grammatical). Bien que les répétitions jouent un rôle essentiel dans le développement du langage (cf. Clark, 2006), nous nous focalisons ici sur les productions spontanées de l'enfant. De ce fait, les répétitions à l'identique de ses propres énoncés (soit 61 énoncés) ou des énoncés de la mère (soit 5 énoncés) seront écartées de l'analyse quantitative et qualitative des différentes formes de référence à soi.

Parmi les formes produites spontanément en position sujet dans des énoncés référant à soi, nous avons relevé :

- des formes conventionnelles : a) le pronom clitique sujet je, b) la combinaison moi je où moi est parfois produit en fin d'énoncé, et c) la structure clivée c'est moi qui, intégrée à nos analyses car la forme moi y 
précède le verbe sans être suivie du clitique je, mais permet, comme moi je, de marquer l'emphase sur le sujet ;

- des syllabes préverbales ou fillers dont la forme et la nature sont encore incertaines, et pouvant être employées seules ou dans des structures $[$ moi + filler+verbe $]$

- des formes non conventionnelles : a) le prénom, également produit par les adultes en interaction avec des enfants, b) le pronom personnel de deuxième personne $t u$, et b) la structure [moi+verbe].

Enfin, tous les énoncés de la mère directement adressés à Anaé et dans lesquels la mère réfère à elle-même en position sujet ont été relevés. Adoptant une approche sémantico-pragmatique, nous analysons également les énoncés de la mère adressés à Anaé et dans lesquels l'enfant est le référent du sujet d'une relation prédicative. Nous présupposons en effet que les formes de référence à soi chez l'enfant peuvent être influencées par la façon dont la mère réfère à elle-même d'une part, et à l'enfant d'autre part.

Afin de rendre compte de l'importance de facteurs sémantiques et pragmatiques, chaque production est ensuite étudiée dans son contexte discursif et interactionnel (sémantisme du verbe avec lequel elle est employée ; mention préalable du référent ; valeur pragmatique de l'énoncé ; participation d'autres personnes à l'interaction).

\subsection{La référence à soi et à l'interlocuteur dans les productions \\ d'Anaé et de sa mère : analyses préliminaires}

Nos analyses préliminaires comparent la production d'énoncés prédicatifs référant à soi et à l'interlocuteur (avec ou sans sujet grammatical) par rapport au total des énoncés produits par la mère et par l'enfant (cf. Figure 1). Malgré 
de légères variations d'un enregistrement à l'autre, on constate tout d'abord qu'en moyenne, sur la totalité des données, Anaé réfère à elle-même dans 5\% de ses énoncés et à sa mère dans $1 \%$ de ses énoncés (cf. Figure 1 ). La mère quant à elle réfère à elle-même dans $6 \%$ de ses énoncés, et à l'enfant dans $23 \%$ de ses énoncés. Ainsi, la référence à soi chez la mère et chez l'enfant représente à peu près la même part des énoncés, tandis que la référence à l'autre est beaucoup plus importante chez la mère.

Figure 1

Dans cette dyade, une grande part de l'interaction s'organise donc autour de l'enfant. Ceci confirme l'hypothèse selon laquelle l'influence de l'input ne peut être appréhendée uniquement par le prisme de la référence à soi chez l'adulte, quantitativement beaucoup moins importante que la référence à l'enfant.

\section{LA REFERENCE A SOI DANS LES PRODUCTIONS D'ANAE}

Dans les analyses présentées ci-dessous, nous examinons dans un premier temps le rapport entre présence et absence de forme de référence à soi dans les productions d'Anaé (3.1). Dans un deuxième temps, nous situons l'emploi de formes non conventionnelles par rapport aux formes conventionnelles (3.2). Enfin, nous étudions les contextes de production des structures non conventionnelles [moi+verbe] (3.2.1) et [prénom+verbe] (3.2.2), en regard avec leur emploi dans le langage de la mère. Le pronom de deuxième personne tu n'étant produit qu'une seule fois sur l'ensemble du corpus dans l'énoncé «tu peux tourner la page ?» (énoncé d'abord adressé à maintes reprises à la mère puis produit très machinalement quand Anaé elle-même 
tourne la page), il n'est pas analysé plus en détail ici. Les résultats sont discutés au fur et à mesure des analyses.

\subsection{Présence et absence de forme de référence à soi chez Anaé}

La première occurrence d'énoncé référant à soi apparaît à 1;06 chez Anaé, avec un prédicat sans sujet (cf. Figure 2). A 1;07, un premier sujet grammatical est produit mais ce n'est qu'à 1;09 que les occurrences d'énoncés référant à elle-même augmentent (17 occurrences). Entre 1;09 et $2 ; 04$, les prédicats avec sujet grammatical représentent en moyenne $40 \%$ des énoncés référant à l'enfant, avec une forte augmentation à $2 ; 00$ où ils représentent plus de $80 \%$ de ces énoncés. Enfin, à partir de 2;05, 90\% en moyenne des verbes référant à l'enfant sont produits avec un sujet grammatical. Le système d'Anaé ressemble alors de plus en plus au système observé dans le langage adulte adressé aux enfants, dans lequel 99\% des prédicats à l'indicatif et au subjonctif sont produits avec un sujet grammatical (Morgenstern et Parisse, 2010). L'expression de la référence à soi en position sujet se met donc en place par paliers successifs.

\section{Figure 2}

L'analyse des contextes éclaire la production ou non de sujet grammatical dans des énoncés référant à soi. Entre 1;07 et 1;10, parmi les 13 occurrences de production du sujet grammatical, 6 correspondent à des situations où l'attention de la mère est détournée par les frères d'Anaé (exemple (1)) et 6 au marquage de son positionnement intersubjectif (cf. Morgenstern, 1994, 2006).

(1) : Anaé $(1 ; 10)$ joue à un jeu avec sa mère et son frère.

Frère: qu'est-ce qu' y a marqué ? (s'adresse à la mère) 

Mère: $\quad$ les $+\ldots$
(s'adresse au frère)
Frère: les bougies et là c'est quoi ? (s'adresse à la mère)
Mère: bougies. (s'adresse au frère)
Anaé: $\quad[\mathbf{e}]$ mets. [e] mets moi $\quad$ (pointe une carte).

A cette période, seul un énoncé référant à elle-même avec un sujet grammatical est produit spontanément par Anaé, sans qu'il s'agisse d'une demande d'attention ou d'un marquage de positionnement intersubjectif. Il s'agit du premier énoncé produit à 1;07 lorsqu'Anaé exprime sa difficulté à assembler des objets et dit «ah [di] peux pas », où [di] a été analysé comme un filler préverbal. Dans tous les autres cas (soit 16 occurrences), lorsque l'attention de la mère n'est pas détournée, que le référent a déjà été mentionné et/ou qu'Anaé n'exprime pas un positionnement par rapport à l'autre, les prédicats sont produits sans sujet (exemple (2)).

(2) : Anaé $(1 ; 10)$ et sa mère sont toutes les deux dans la cuisine et s'apprêtent à prendre un goûter.

Mère: $\quad$ tu veux un gâteau ? (s'adresse à Anaé)

Anaé: $\quad$ veux ça. (pointe un paquet de gâteaux)

Ainsi, entre $1 ; 07$ et $1 ; 10$, le sémantisme du verbe ne semble pas avoir d'impact sur la production ou non d'un sujet. En effet, les structures [sujet+verbe] sont produites avec 9 verbes différents, dont des verbes d'action comme mettre, porter, sauter, marcher ou des modaux comme vouloir et pouvoir. Les prédicats sans sujet, quant à eux, correspondent à 8 verbes différents, dont également des verbes d'action comme enlever, appuyer, porter, monter, de sentiment comme avoir peur, d'état comme être ou des 
modaux comme vouloir. En outre, tous les énoncés faisant référence à soi, qu'ils soient produits avec un sujet ou non, servent l'expression d'un désir.

L'expression de la référence à soi avec un sujet grammatical dépend donc avant tout des intentions communicatives d'Anaé dans l'interaction : lorsque l'accessibilité du référent par l'adulte est contrariée (cf. Serratrice, 2005) car son attention est détournée, ou que l'enfant exprime une distance entre soi et l'autre, un sujet grammatical est produit; lorsque la « consensualité » avec l'adulte (Morgenstern, $2006: 12)$ est maintenue, le prédicat est produit sans sujet.

A 2 ans, il devient plus difficile de distinguer un système aussi régulier. Il semblerait qu'il s'agisse d'une période de transition vers le système adulte, tant du point de vue quantitatif que qualitatif : le nombre de prédicats référant à soi augmente très fortement, la proportion d'énoncés avec sujet passe de moins de $50 \%$ à plus de $80 \%$ (bien plus que dans les trois enregistrements suivants) et les critères de description du système utilisés précédemment ne permettent que difficilement de rendre compte du système de l'enfant à cet âge.

Après 2 ans, le système d'Anaé se stabilise à nouveau. L'emploi de prédicats sans sujet est alors essentiellement observé avec les verbes vouloir, pouvoir, savoir ou l'auxiliaire aller. Ainsi, parmi les 45 occurrences de prédicats sans sujet entre 2;01 et 3;00,39 occurrences correspondent à l'un de ces 4 verbes alors qu'Anaé produit près de 30 verbes différents pendant cette période. Il semblerait donc qu'Anaé reconstruise, à une période où un grand nombre de verbes est produit dans des constructions syntaxiques 
[sujet+verbe], un paradigme de verbes à forte valeur modale pour lesquels le référent sujet peut être implicite.

Les verbes de ce paradigme peuvent néanmoins parfois être produits avec un sujet grammatical. Entre $2 ; 01$ et $2 ; 04$, environ $30 \%$ sont produits avec un sujet grammatical dans des conditions similaires à celles identifiées plus tôt: alors que la référence à soi tend à ne pas être marquée linguistiquement, elle l'est dans les cas où l'attention de la mère est détournée ou lorsqu'Anaé marque son positionnement vis-à-vis de l'adulte. A partir de 2;05, cette opposition sémantique et fonctionnelle s'estompe progressivement : environ $90 \%$ de ces prédicats à valeur modale sont produits avec un sujet grammatical, quelle que soit la situation d'interaction.

Après 2 ans, Anaé entre donc dans le système de la langue et de ses locuteurs : une majorité de verbes référant à elle-même est produite avec un sujet grammatical. Mais si les structures [sujet+verbe] sont rapidement prédominantes dans les énoncés d'Anaé référant à elle-même, une variété de formes remplit la position sujet, dont des formes grammaticalement non conventionnelles. Nous montrons dans la partie suivante que ces formes non conventionnelles, qui restent relativement peu fréquentes (3.2), sont produites dans des contextes interactionnels spécifiques, et que l'association entre forme et fonction pragmatique peut être retracée dans les productions de la mère (3.2.1 et 3.2.2).

\subsection{Formes conventionnelles et non conventionnelles de référence à soi chez Anaé}

On distingue trois périodes dans le développement des formes de référence à soi en position sujet chez Anaé (cf. Figure 3). Entre 1;07 et 1;10, les quelques 
formes produites sont majoritairement des fillers (10 occurrences entre 1;07 et $1 ; 10$ ) bien que 3 occurrences de formes grammaticalement non conventionnelles soient produites. A partir de 2 ans, les sujets grammaticaux sont plus nombreux, les formes conventionnelles apparaissent et leur proportion augmente progressivement, tandis que la production de fillers diminue fortement. La proportion des formes non conventionnelles varie mais représente en moyenne $10 \%$ des formes produites entre $2 ; 00$ et $2 ; 08$. Entre $2 ; 10$ et 3;00, les formes conventionnelles représentent plus de $90 \%$ des sujets. Les fillers sont encore présents mais peu fréquents et les formes non conventionnelles ont disparu.

\section{Figure 3}

Un développement par paliers successifs est donc observable à la fois dans le développement de la structure [sujet+verbe] et dans le développement de la nature de ces sujets, développement par étapes également observés dans les études de Brigaudiot et al. (1994) et Morgenstern (1995, 2006). Comme dans les études précédentes, on observe chez Anaé que les formes conventionnelles, les fillers et les formes non conventionnelles sont concomitants pendant une période de 12 mois. Pourquoi l'enfant produit-elle des formes non conventionnelles alors qu'elle est en mesure de produire des formes conventionnelles ? Dans ce qui suit, nous montrons que la présence de ces formes dépend à la fois des stratégies communicatives employées par l'enfant pour répondre à certains facteurs sémantiques et pragmatiques, et du langage qui lui est adressé par la mère.

\subsubsection{Usage des structures avec moi chez Anaé et influence de l'input}


Parmi les formes non conventionnelles de référence à soi, on distingue la structure $[m o i+$ verbe]. Bien que moi soit l'un des mots les plus fréquemment produits par Anaé autour de 2 ans (Leroy-Collombel et Morgenstern, ce numéro), cette structure ne représente en moyenne, sur la totalité des données, que $5 \%$ des formes de référence à soi en position sujet (cf. Tableau 1).

\section{Insérer le Tableau 1 par ici}

L'analyse des énoncés dans lesquels la structure [moi+verbe] est produite révèle qu'Anaé ne les emploie qu'avec les verbes vouloir, savoir et l'auxiliaire aller. Il semblerait donc que la structure [moi+verbe] soit appliquée aux mêmes prédicats que ceux pouvant par ailleurs référer à soi sans sujet grammatical (cf. 3.1). Puis à partir de 2;05, ces verbes à valeur modale sont intégrés au système des autres verbes, et sont alors également produits dans des structures [je+verbe] ou $[m o i+j e+$ verbe $]$.

L'analyse des contextes interactionnels dans lesquels les structures $[m o i+j e / f i l l e r+v e r b e]$ et $[m o i+v e r b e]$ sont produites suggère que l'emploi de moi dépend de facteurs pragmatiques: moi est toujours utilisé dans des contextes de positionnement par rapport à l'autre, d'opposition ou de comparaison, comme c'est le cas dans l'exemple (3).

(3) : Anaé $(1 ; 10)$ et son frère sont prêts à prendre le goûter dans la cuisine.

Frère: $\quad$ moi $\mathbf{j}(\mathbf{e})$ veux boire de l'eau.

Mère: $\quad$ attends $j(e)$ vais en chercher.

Anaé: $\quad$ moi veux de l'eau.

Ainsi, moi vient ajouter un marqueur de positionnement intersubjectif aux prédicats qui sont produits avec ou sans sujet grammatical dans des 
contextes non marqués, comme cela a pu être observé dans les études de Brigaudiot et al. (1994) et Morgenstern (1994, 2006).

Cette association du marqueur moi à une fonction pragmatique de positionnement vis-à-vis de l'autre trouve-t-elle son origine dans le langage de la mère ? En référence à soi et à l'enfant, les pronoms personnels clitiques sujets je et $t u$ représentent en moyenne $92 \%$ et $97 \%$ respectivement des formes produites en position sujet par la mère (cf. Figure 4). La prédominance de ces formes dans le langage de la mère joue donc sans doute un rôle primordial dans la reconstruction et la production précoce et fréquente des structures [je/filler+verbe] par Anaé (cf. Tableau 1). Mais la mère emploie également d'autres formes de référence à soi et à l'enfant, plus sporadiques : moi je ou toi tu, c'est toi/moi qui, ainsi que des combinaisons avec une troisième personne comme maman elle ou Anaé elle.

\section{Figure 4}

L'analyse des contextes dans lesquels ces formes sont produites par la mère montre que leur emploi repose sur des paramètres pragmatiques. Les structures clivées c'est moi/toi qui sont produites lorsque la mère individualise un référent, marquant alors de manière explicite une opposition avec d'autres référents possibles (comme dans « non c'est pas toi qui l'as fait c'est Ael qui a dessiné les stroumpfs !»-Anaé 1;06) ou implicite («l'huile c'est moi qui vais la mettre pour pas qu'on en renverse partout d'accord ?» Anaé 2;11). Les combinaisons moi je et toi tu sont souvent employées conjointement et marquent une distribution des rôles agentifs entre la mère et l'enfant comme dans «allez, moi j(e) lui fais un câlin et toi tu fais manger Kirikou, d'accord?» (Anaé 2;01). Bien que ces structures soient peu 
fréquentes, la saillance des contextes dans lesquels la mère les emploie pourrait faciliter leur acquisition et expliquer leur rapide spécialisation dans le marquage du positionnement intersubjectif chez l'enfant.

\subsubsection{Usage du prénom chez Anaé et rôle de l'input}

Parmi les formes non conventionnelles qu'Anaé produit, on note également l'emploi du prénom et de la troisième personne. Bien qu'elle entende qu'on réfère à elle à la troisième personne dans les échanges entre les membres de sa famille, et bien qu'elle soit régulièrement interpelée par son prénom (on observe 548 vocatifs avec le prénom d'Anaé dans nos enregistrements entre 1;05 et 3;00, soit en moyenne 35 occurrences par heure), les usages de son propre prénom par Anaé sont rares et ne représentent que 4\% des sujets grammaticaux en moyenne (cf. Tableau 1). Ce n'est donc pas la simple fréquence de cette forme dans l'input, dont le référent est pourtant unique, qui influence sa production par Anaé.

A la lumière des analyses de Morgenstern $(1992,2006)$, on peut analyser les usages de la troisième personne et de son prénom par Anaé comme spécifiques au récit d'événements marquants. Ainsi, à 2;04, Anaé reprend un énoncé de sa mère pour redire qu'elle, Anaé, a pris le train (exemple (4)).

(4) : Anaé $(2 ; 04)$ et sa mère lisent un livre qui les conduit à reparler d'une sortie au zoo.

Mère: $\quad$ oui tu $t(\mathrm{e})$ rappelles quand on était allés au zoo ?

Anaé: $\quad$ ouais.

Mère: on avait pris le train.

Anaé: $\quad$ Anaé [e] a pris [o] train ! 
Mère: Anaé elle a pris le train et on est allés où ?

L'utilisation de la troisième personne dans le récit suggère également une distanciation entre le soi passé et le soi présent de l'enfant qui remet en mots cette expérience (Morgenstern, 2006, 2009). Cet énoncé ainsi que l'usage de la troisième personne sont repris par la mère, qui unifie l'expérience passée de l'enfant à une expérience commune de personnes coprésentes dans la situation actuelle avec « on ».

Dans les productions spontanées de la mère (non reprises des productions de l'enfant), ces usages sont sporadiques, mais ils correspondent également toujours à la description de situations extraordinaires (exemple (5)) ou d'événements inhabituels ou marquants de la vie de l'enfant, comme le fait de ne plus mettre de couches (exemple (6)).

(5) : Anaé $(1 ; 10)$ est sur son toboggan mais elle n'ose pas se laisser glisser.

Mère: oh lala qu'est-c(e) qu'elle est grande Anaé !

Mère: bah voilà très bien ! (Anaé monte les marches toute seule).

Anaé: $\quad 0$ (fait des petits bruits et tourne la tête vers la mère)

Mère: $\quad$ qu' est-c(e) qu' y a ?

Anaé: [3] va tomber Anaé.

Mère: $\quad$ mais non elle va pas tomber Anaé ! je te tiens (rit)!

(6) : Anaé $(3 ; 0)$ raconte qu'elle pleurait quand elle était petite. Sa mère évoque alors l'achat d'un poupon qui pleure.

Mère : et alors, pourquoi maman elle t'a acheté le bébé qui pleure?

Anaé : parce que ai envie de jouer avec. 
Mère : non. c'est pas pour ça . c'était parce que maintenant tu es une...

Anaé: ... grande.

Mère: et pourquoi tu es une grande?

Anaé : parce que ... je sais pas.

Mère: ben parce que tu mets plus de ...

Anaé: ... couche.

L'intonation et d'autres marqueurs emphatiques renforcent le caractère exceptionnel de l'événement et participent à la mise à distance entre le référent et l'interlocuteur. Comme dans l'exemple (4), cet événement est ensuite replacé dans la situation présente et la référence à soi et à l'enfant est à nouveau exprimée avec les première et deuxième personnes.

A partir du langage de la mère, il est donc possible que l'enfant intègre une association entre l'emploi du prénom et des contextes d'utilisation spécifiques, comme cela semble également être le cas pour la forme moi. Puis peu à peu, à partir de 2;05 notamment, le système d'Anaé se grammaticalise. Tandis que les verbes à valeur modale sont eux-mêmes de plus en plus souvent produits avec un sujet et que la référence à soi est presque toujours marquée avec un sujet grammatical indépendamment du contexte, les formes non conventionnelles disparaissent. Les fonctions qu'elles exprimaient sont remplies par les formes conventionnelles accompagnées d'autres marqueurs linguistiques, comme le marqueur d'opposition «mais » ou les marqueurs d'argumentation (cf. Sekali, ce numéro).

\section{CONCLUSION}

Nous avons montré dans cette étude que le développement de la référence à soi chez l'enfant, et en particulier des formes non conventionnelles en 
position sujet, devait être appréhendé à la fois par le prisme de ses propres intentions communicatives (cf. Brigaudiot et al., 1994 ; Morgenstern, 1995, 2006) et par celui de l'influence du langage adressé par les parents (Budwig, 1996).

Ce croisement des approches fonctionnalistes et usage-based nous a ainsi permis de mettre en évidence deux phénomènes dans les productions d'Anaé entre 1;05 et 3;00. Concernant tout d'abord la production ou non de sujet grammatical, nos analyses montrent qu'avant 2 ans, celle-ci dépend essentiellement du contexte interactionnel dans lequel le prédicat est produit. Après 2 ans, Anaé reconstruit un paradigme de verbes à valeur modale (comme vouloir, pouvoir, aller+verbe, exprimant la volonté, la possibilité ou le projet) pour lesquels la référence à soi peut être implicite. La production d'un sujet grammatical dépend alors à la fois de facteurs sémantiques et pragmatiques. Ces analyses illustrent parfaitement la reconstruction active du système par l'enfant telle qu'elle est mise en évidence dans les approches fonctionnalistes. De même, l'étude des contextes dans lesquels moi et le prénom sont produits montre que ces formes sont produites dans des contextes interactionnels spécifiques. Moi marque l'opposition, la comparaison, le positionnement intersubjectif, et c'est la valeur sémantique du verbe qui détermine l'emploi de la structure [moi+verbe] ou $[m o i+j e /$ filler+verbe $]:$ les verbes à valeur modale, qui ne prennent pas de sujet grammatical par ailleurs, sont produits dans la structure [moi+verbe] quand les énoncés marquent le positionnement interactionnel. Le prénom est employé dans des récits d'événements extraordinaires et marquants. Ces analyses longitudinales et en contexte confirment donc les observations faites 
chez d'autres enfants francophones par Brigaudiot et al. (1994) et Morgenstern (1992, 2006).

Cependant, une analyse fonctionnelle des usages des structures avec moi ou toi et du prénom par la mère montre qu'elle-même emploie ces formes dans des contextes interactionnels comparables. La recréation par l'enfant d'une association entre ces formes et leurs contextes d'utilisation émane donc probablement de la présence de telles associations dans l'input. Ces données font écho à l'étude de Budwig (1996) sur l'usage de [I+verbe] avec des référents non agentifs chez des enfants anglophones et leurs parents, ainsi qu'à l'étude de Morgenstern (2011) sur l'emploi du prénom dans une dyade mère-enfant.

Nos résultats et analyses sur les données d'Anaé montrent en outre que les productions de l'enfant ne sont pas uniquement influencées par la fréquence des formes ou des structures présentes dans l'input, comme cela a pu être proposé par Kirjavainen et al. (2009), mais qu'elles le sont également par les usages de ces formes en interaction, leur valeur sémantique et pragmatique. La saillance des contextes dans lesquels ces formes sont employées par les adultes accentue probablement leur fonction pragmatique spécifique, ce à quoi les enfants sont particulièrement sensibles lors du processus d'acquisition du langage (Clark et Clark, 1979). D'autres études du développement de la référence à soi chez l'enfant francophone dans lesquelles serait analysée de manière systématique l'influence des contextes sémantiques et pragmatiques d'une part, et de l'input d'autre part sont à présent nécessaires. La mise en regard de ces données permettra ainsi de mieux rendre compte de l'existence d'une corrélation entre les 
caractéristiques à la fois formelles et fonctionnelles de l'input et des proportions et usages de formes non conventionnelles dans le langage de l'enfant. Cette mise en regard de données quantitatives et qualitatives fines d'enfants en acquisition d'une même langue permettra également de mettre en évidence la singularité de chaque enfant dans ce qui pour lui, à une période donnée, fait perceptuellement et fonctionnellement sens dans la mise en mots du soi.

REMERCIEMENTS Je remercie tout particulièrement Aliyah Morgenstern, Martine Sekali, Marie Leroy-Collombel, Karine Martel, Marion Blondel, Christophe Parisse, Caroline Rossi, Camelia Dascalu et deux relecteurs anonymes pour leurs critiques constructives et leurs suggestions avisées.

Nombre total de mots (titre, abstract, mots clés, article, remerciements, références, titres de graphiques) : 6393

Date de remise du manuscrit révisé : 10 août 2011

\section{RÉFÉRENCES}

Brigaudiot, B., Morgenstern, A. et Nicolas, C. (1994). 'Me found it, I find it'. A la recherche de 'je' entre deux et trois ans. Faits de langue - La personne, $3: 123$ 131.

Budwig, N. (1995). A developmental-functionalist approach to child language. NewJersey: Erlbaum.

Budwig, N. (1996). What influences children's patterning of forms and functions in early child language? In : D. Slobin, J. Gerhardt, A. Kyratzis et G. Jiansheng 
(eds), Social interaction, social context and language: Essays in honour of Susan Ervin-Tripp. Mahwah, NJ: Lawrence Erlbaum, pp. 143-156.

Chiat, S. (1986). Personal pronouns, in P. Fletcher et M. Garman (eds), Language acquisition. Cambridge: Cambridge University Press. 2nd edition, pp.339-355.

Chiat, S. (1981). Context-specificity and generalization in the acquisition of pronominal distinctions. Journal of Child Language, 8: 75-91.

Clark, E.V. (2006). La répétition et l'acquisition du langage. La linguistique 42(2): $67-79$

Clark, E.V. et Clark, H.H. (1979). When nouns surface as verbs. Language 55: 767811.

Charney, R. (1980). Speech roles and the development of personal pronouns. Journal of Child Language, 7: 509-528.

Gadet, F. (1989). Le français ordinaire. Paris: Colin.

Gerken, L. (1994). Young Children's Representation of Prosodie Phonology: Evidence from English-Speakers' Weak Syllable Productions. Journal of Memory and Language, 33: 19-38.

Kirjavainen, M., Theakston, A. et Lieven, E. (2009). Can input explain children's mefor-I errors? Journal of Child Language, 36: 1091-1114.

Leroy-Collombel, M. et Morgenstern, A. (2012). Rising grammatical awareness in a French-speaking child from 18 to 36 months: uses and misuses of possession markers. Journal of French Language Studies, this issue, Cambridge: Cambridge University Press.

Mahler, M. S., F. Pine et A. Bergman. (1975). The psychological birth of the human infant: Symbiosis and individuation. New York: Basic.

Morgenstern, A. (1995). L'enfant apprenti-énonciateur. L'auto-désignation chez 
l'enfant en français, en anglais et en langue des signes française. Thèse non publiée. Université Paris 3 - Sorbonne nouvelle.

Morgenstern, A. (1996). Pour qui JE parle. Groupe T.E.L.O.S, La détermination, Presses de la Sorbonne Nouvelle, pp. 105-128.

Morgenstern, A. (2003). Le langage de l'enfant est-il linguistiquement correct ? Correct, incorrect en linguistique anglaise, CIEREC Travaux 113. Numéro dirigé par Claude Delmas. Publications de l'Université de St Etienne. pp. 111128.

Morgenstern, A. (2006). Un JE en construction. Ontogenèse de l'auto-désignation chez l'enfant. Paris : Bibliothèque de Faits de langues, Ophrys.

Morgenstern, A. (2007), Discours repris, discours emprunté, discours habité chez l'enfant entre 1 et 3 ans. Revista do Gel, 04 (2) : 171-188.

Morgenstern, A. (2009). L'enfant dans la langue. De l'observation du naturaliste à l'analyse du linguiste. Paris : Presses de la Sorbonne Nouvelle.

Morgenstern, A. et Brigaudiot, M. (2004). L'altérité dans l'identité : quand l'enfant parle de lui à la deuxième personne. Identité(s) : 183-193

Morgenstern, A. et Parisse, C. (2010). Constructing "basic" verbal constructions: a longitudinal study of the blossoming of constructions with six frequent French verbs. 3rd UK Cognitive Linguistics Conference. Hatfield, UK, July 2010.

Morgenstern, A. (2011). Autoréférence et variations dans des dialogues mère-enfant. Actes du colloque Res per Nomen, Pierre Frath (Ed.), Université de Reims.

Ninio, A. et Snow, C.E. (1996). Pragmatic development. Essays in developmental science. Boulder, CO, US: Westview Press. xii, 222 pp.

Oshima-Takane, Y., Goodz, E. et Derevensky, J.L. (1996). Birth Order Effects on Early Language Development: Do Second-born Children Learn from Overheard 
Speech? Child Development, 67(2): 621-634.

Peters, A. et Menn, L. (1993). False starts and filler syllables: ways to learn grammatical morphemes. Language, 69 (4):742-777.

Pettito, L.A. (1987). On the autonomy of language and gesture: Evidence from the acquisition of personal pronouns in American Sign Language. Cognition, 27(1): $1-52$.

Rabain-Jamin, J. et Sabeau-Jouannet, E. (1989). Playing with pronouns in French maternal speech to prelingual infants. Journal of Child Language, 16: 217-238.

Rispoli, M. (2005). When children reach beyond their grasp: why some children make pronoun case errors and others don't. Journal of Child Language, 32: 96113.

Sekali, M. (2012). The emergence of complex sentences in a French child's language from $0 ; 10$ to 4;01: causal adverbial clauses and the concertina effect. Journal of French Language Studies, this issue, Cambridge: Cambridge University Press.

Serratrice, L. (2005). The role of discourse pragmatics in the acquisition of subjects in Italian. Applied Psycholinguistics, 26: 437-462.

Tomasello, M. (2003). Constructing a language, a usage-based theory of language acquisition. Cambridge: Harvard University Press.

Wills, D.D. (1977). Participant deixis in English and baby talk. In : C.E. Snow et C.A. Ferguson (eds), Talking to children : language input and acquisition. Cambridge: Cambridge University Press, pp. 271-298.

Zazzo, R. (1948). Image du corps et conscience de soi. Enfance, $1: 29-43$. 


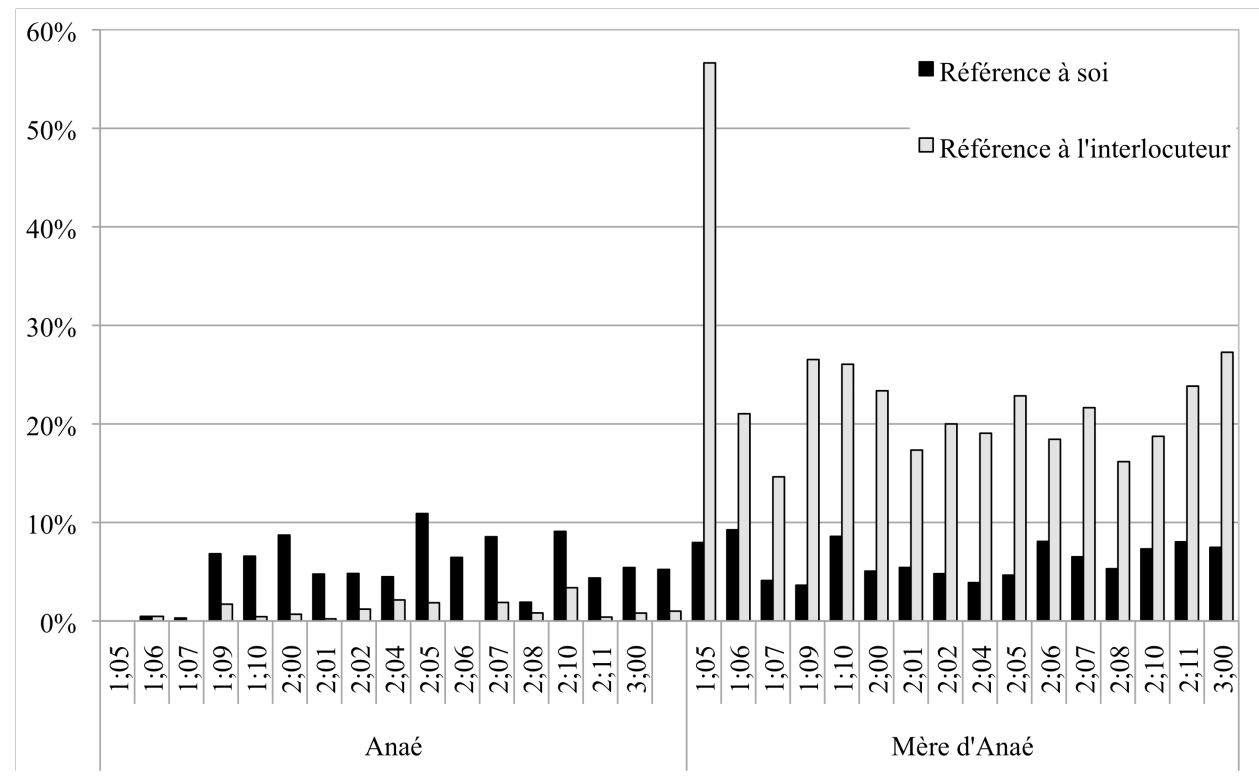

Figure 1 : Proportion de la référence à soi et la référence à l'interlocuteur en position sujet par rapport au total d'énoncés chez Anaé et sa mère en fonction de l'âge d'Anaé. 


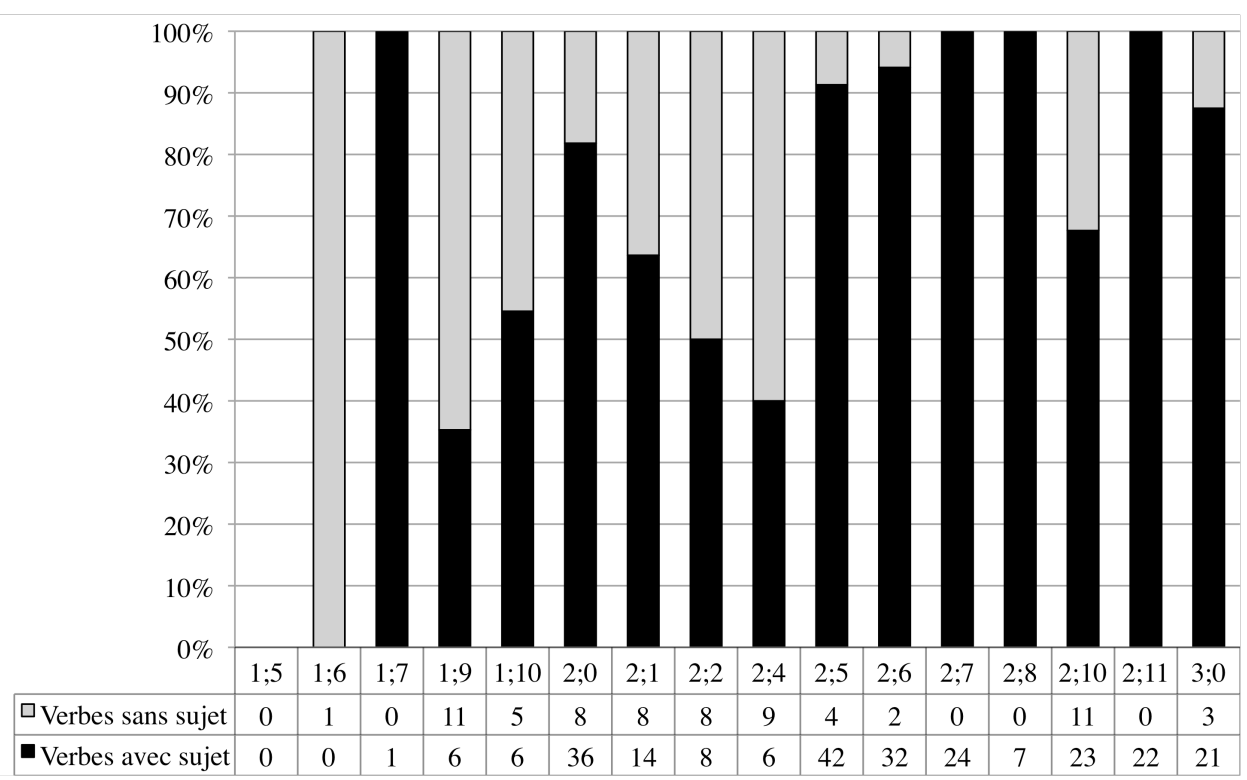

Figure 2 : Proportion et fréquences des verbes avec et sans sujet grammatical dans les énoncés d'Anaé en fonction de son âge. 


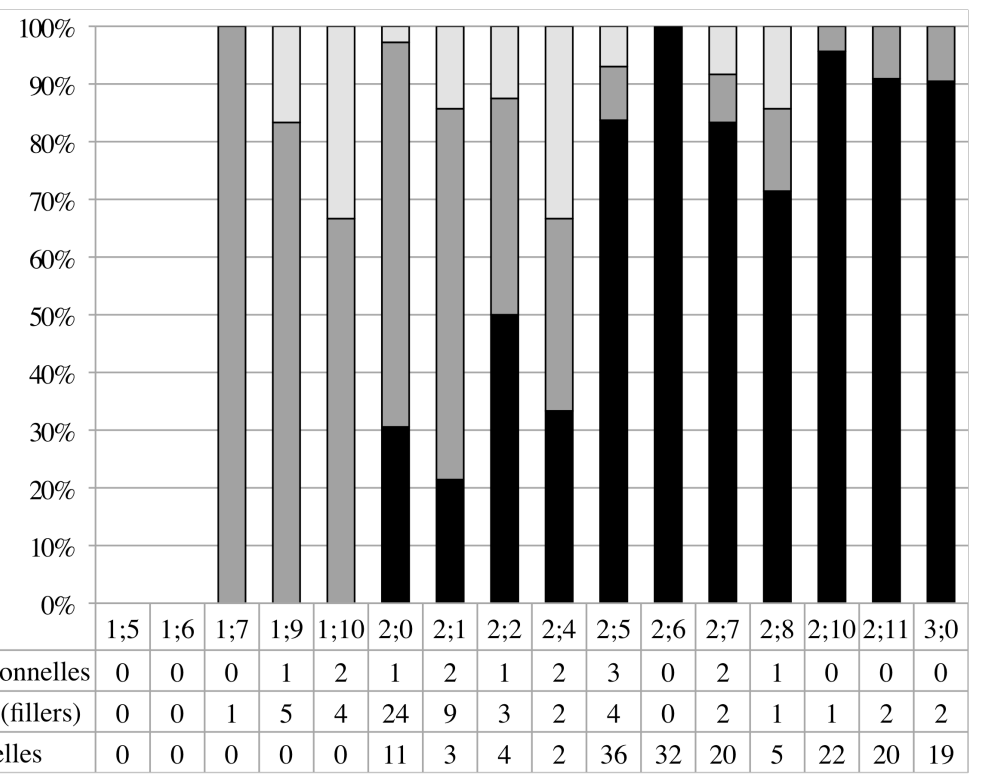

Figure 3 : Proportion et fréquences des formes conventionnelles, syllabes préverbales (fillers) et formes non conventionnelles de référence à soi chez Anaé en fonction de son âge. 


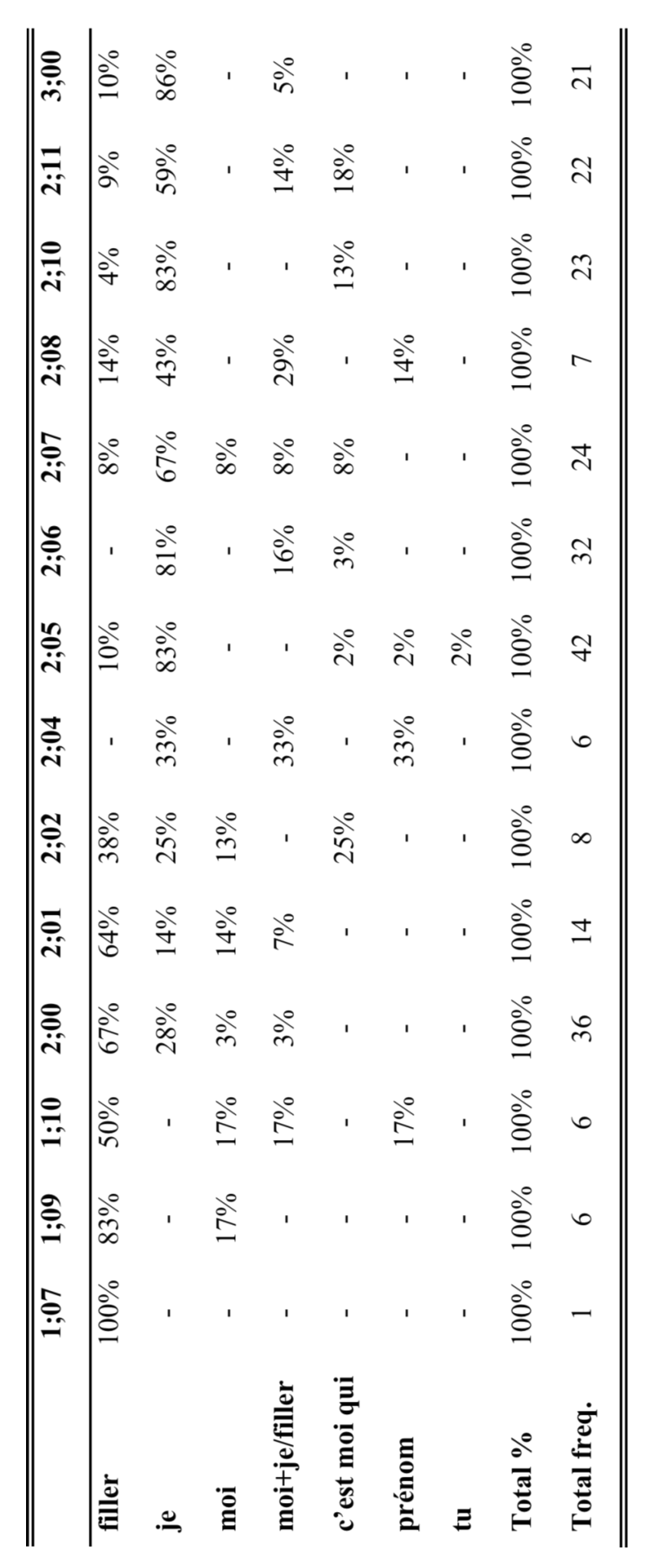

Tableau 1 : Proportion de chaque forme de référence à soi par rapport au total des formes chez Anaé en fonction de son âge. 


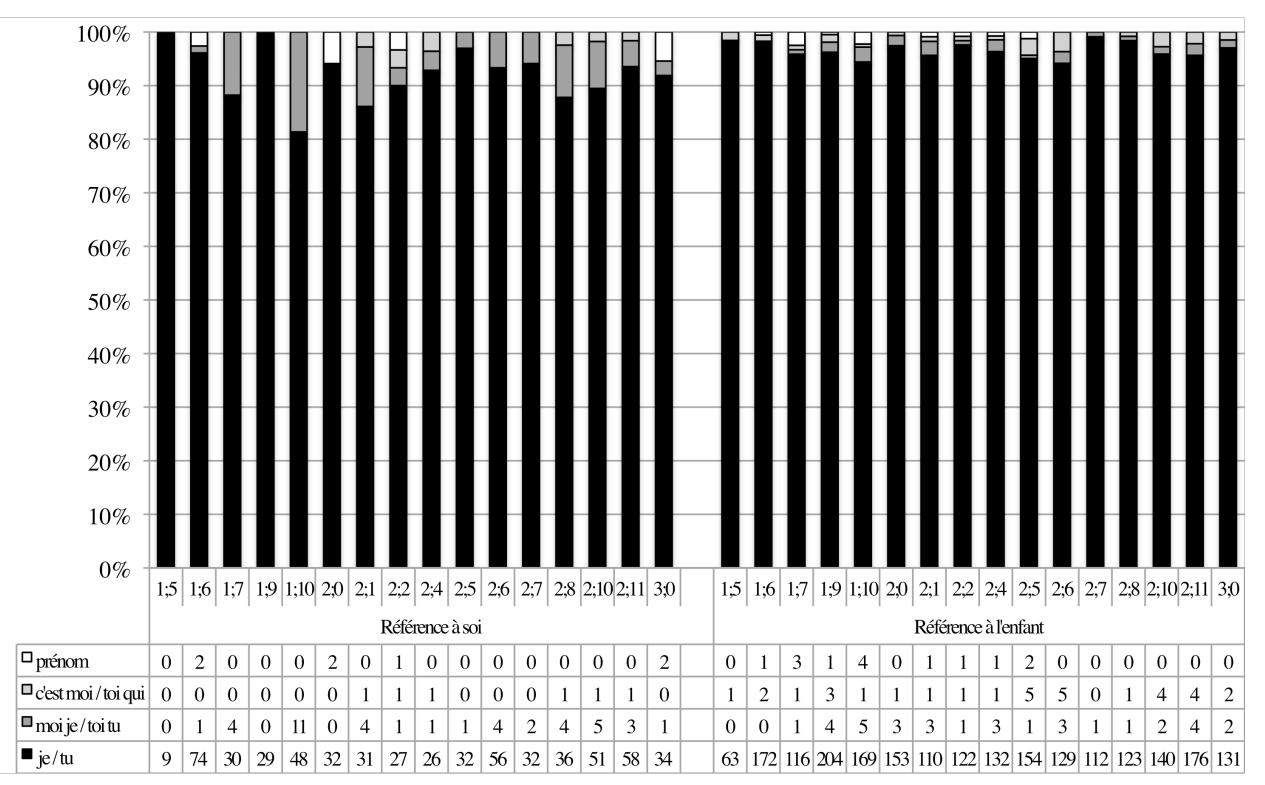

Figure 4. Proportion et fréquences des formes de référence à soi et à l'enfant chez la mère en fonction de l'âge d'Anaé. 